\title{
ACKNOWLEDGMENT OF REVIEWERS
}

The success of Molecular Plant-Microbe Interactions depends on the quality of manuscripts submitted by authors and on the care and competence with which they are reviewed. It is the policy of the Editorial Board to solicit reviews of manuscripts from specialists most qualified to review them. In addition to members of the Editorial Board, the individuals listed below provided constructive critical reviews of one or more manuscripts during the past year. Their names are published here in grateful appreciation for their contributions to the journal.

F. Allen, University of Tennessee, Knoxville, TN, U.S.A.

R. Allison, Michigan State University, East Lansing, MI U.S.A.

P. Apel-Birkhold, USDA-ARS, PSI, BPDL, Beltsville, MD U.S.A.

M. Arlat, CNRS-INRA, Castanet-Tolosan, France

C. J. Baker, USDA, MPPL, BARC-WEST, Beltsville, MD, U.S.A.

H. Barker, Scottish Crop Research Institute, Invergowie, Dundee, Scotland

S. J. Barker, University of Adelaide, Waite Osmond, Australia

F. Barras, LCB - CNRS, Marseille, France

W. Barz, Universitat Munster, Munster/Westfalische, Germany

D. L. Beck, Mt. Albert Research Centre, Auckland, New Zealand

S. V. Beer, Cornell University, Ithaca, NY, U.S.A.

C. Bender, Oklahoma State University, Stillwater, OK, U.S.A.

A. Bent, University of Illinois, Urbana, IL, U.S.A.

S. Bereswill, Inst. f. Medizinische Mikrobiologie und Hygiene, Freiburg, Germany

H. Bertrand, Michigan State University, East Lansing, MI, U.S.A.

D. M. Bird, North Carolina State University, Raleigh, NC, U.S.A.

D. Bisaro, Ohio State University, Columbus, OH, U.S.A.

T. Bisseling, Wageningen Agricultural University, Wageningen, The Netherlands

D. Blevins, University of Missouri, Columbus, MO, U.S.A.

G. Boccardo, Istituto di Fitovirologia Applicata, Torino, Italy

P. Bonfante, Universita Torino, Torino, Italy

R. Bostock, University of California, Davis, CA, U.S.A.

P. Bottomley, Oregon State University, Corvallis, OR, U.S.A.

R. Bressan, Purdue University, West Lafayette, IN, U.S.A.

N. Brewin, John Innes Institute, Norwich, United Kingdom

W. Broughton, Universite de Geneve, Geneve, Switzerland

G. Bryan, Scottish Crop Research Institute, Invergowie, Dundee, Scotland

R. Buell, Carnegie Institute, Stanford, CA, U.S.A.

J. J. Bujarski, Northern Illinois University, De Kalb, IL, U.S.A.

T. Burr, Brookhaven National Laboratory, Upton, NY, U.S.A.

R. Cameron, University of Toronto, Toronto, Canada

R. Carlson, University of Georgia, Athens, GA, U.S.A.

J. P. Carr, University of Cambridge, Cambridge, United Kingdom

S. Chapman, Scottish Crop Research Institute, Invergowie, Dundee, Scotland

A. Chatterjee, University of Missouri, Columbia, MO, U.S.A.

Z. Chen, Univeristy of Idaho, Moscow, ID, U.S.A.

P. Christie, University of Texas Health Science Center, Houston, TX, U.S.A.

A. Collmer, Cornell University, Ithaca, NY, U.S.A.

C. W. Collmer, Cornell University, Ithaca, NY, U.S.A.

D. A. Cooksey, University of California-Riverside, Riverside,
CA, U.S.A.

D. Coplin, Ohio State University, Columbus, OH, U.S.A.

N. Crawford, University of California, San Diego, La Jolla, CA, U.S.A.

P. Cregan, BARC-WEST, Beltsville, MD, U.S.A.

M. Crespi, CNRS Institut Science Vegetale, Gif-sur-Yvette Cedex, France

M.-J. Daboussi, Institut de Genetique et Microbiologie, Orsay, Cedex, France

T. Dalmay, John Innes Centre, Norwich, England

M. Daniels, John Innes Centre, Norwich, England

A. Das, University of Minnesota, St. Paul, MN, U.S.A.

K. Davis, Ohio State University, Columbus, OH, U.S.A.

F. Dazzo, Michigan State University, East Lansing, MI, U.S.A.

W. De Jong, Scottish Crop Research Institute, Invergowie, Dundee, Scotland

R. Dean, Clemson University, Clemson, NC, U.S.A.

H. Deising, Universität Konstanz, Konstanz, Germany

T. P. Delaney, Cornell University, Ithaca, NY, U.S.A.

G. DeLorenzo, Universita di Roma "La Sapienza," Roma, Italy

Y. Dessaux, CNRS Institut Science Vegetale, Gif-sur-Yvette, France

M. Dickman, University of Nebraska, Lincoln, NE, U.S.A.

R. Dietrich, University of North Carolina, Chapel Hill, NC, U.S.A.

R. Dietzgen, University of Queensland, St. Lucia, Australia

A. Downie, John Innes Institute, Norwich, United Kingdom

K. Dunn, Boston College, Chestnut Hills, MA, U.S.A.

D. Ebbole, Texas A\&M University, College Station, U.S.A.

A. Ellingboe, University of Wisconsin-Madison, Madison, WI, U.S.A.

J. English, John Innes Centre, Norwich, United Kingdom

N. Everett, Interlink Associates, Auburn, CA, U.S.A.

M. Farman, University of Kentucky, Lexington, KY, U.S.A.

S. Farrand, University of Illinois at Urbana-Champaign, Urbana, IL, U.S.A.

A. Feign, Monell Chemical Senses Center, Philadelphia, PA, U.S.A.

J. Felix, University of Basel, Basel, Switzerland

H.-M. Fischer, Mikrobiologie Institut ETH-Zentrum, Zurich, Switzerland

D. Fulbright, Michigan State University, East Lansing, MI, U.S.A.

W. C. Fuqua, Trinity University, San Antonio, TX, U.S.A.

D. W. Gabriel, University of Florida, Gainesville, FL, U.S.A.

T. Gaffney, Ciba Biotechnology, Research Triangle Park, NC, U.S.A.

J. A. Garcia, Universidad Autonoma, Madrid, Spain

S. Genin, INRA-CNRS, Castanet-Tolsan, France 
D. Gilchrist, University of California, Davis, CA, U.S.A.

R. Goldbach, Wageningen Agricultural University, Wageningen, The Netherlands

J. Golden, Texas A\&M University, College Station, TX, U.S.A.

C. Gonzalez, Texas A\&M University, College Station, TX, U.S.A.

M. Gordon, University of Washington, Seattle, WA, U.S.A.

T. Gordon, University of California, Berkeley, CA, U.S.A.

J. Gorlach, CIBA-Ag. Biotech. Res. Facility, Research Triangle Park, NC, U.S.A.

F. Govers, Wageningen Agricultural University

T. Graham, Ohio State University, Columbus, OH, U.S.A.

S. Gray, Cornell University, Ithaca, NY, U.S.A.

D. Gross, Washington State University, Pullman, WA, U.S.A.

M. L. Guerinot, Dartmouth College, Hanover, NH, U.S.A.

D. Haas, Universite de Lausanne, Lausanne-Dorigney, Switzerland

M. G. Hahn, University of Georgia, Athens, GA, U.S.A.

J. Hamer, Purdue University, West Lafayette, IN, U.S.A.

R. Hammerschmidt, Michigan State University, East Lansing, MI, U.S.A.

J. Handelsman, University of Wisconsin-Madison, Madison, WI, U.S.A.

J. E. Harper, University of Illinois, Urbana, IL, U.S.A.

S. Y. He, Michigan State University, East Lansing, MI, U.S.A.

M. C. Heath, University of Toronto, Toronto, ON, Canada

G. Hernandez, Universidad Nacional Autonoma de Mexico, Cuernavaca, Mor. Mexico

B. Hillman, Rutgers University, New Brunswick, NJ, U.S.A.

Susan Hirano, University of Wisconsin-Madison, Madison, WI, U.S.A.

T. Hoover, University of Georgia, Athens, GA, U.S.A.

P. J. J. Hooykaas, Leiden University, Leiden, The Netherlands

S. Howell, Boyce Thompson Institute, Ithaca, NY, U.S.A.

A. G. Hunt, University of Kentucky, Lexington, KY, U.S.A.

R. Hussey, University of Georgia, Athens, GA, U.S.A.

S. Hutcheson, University of Maryland, College Park, MD, U.S.A.

C. Ishimaru, Colorado State University, Fort Collins, CO, U.S.A.

T. Jack, Datmouth College, Hanover, NH, U.S.A.

J. Jones, John Innes Centre, Norwich, United Kingdom

M. H. A. J. Joosten, Wageningen Agricultural University, Wageningen, The Netherlands

C. I. Kado, University of California, Davis, CA, U.S.A.

R. Kahmann, Institut für Genetik und Mikrobiologie, Muenchen, Germany

S. Kamoun, Agricultural University, Wageningen, The Netherlands

J. Kemp, New Mexico State University, Las Cruces, NM, U.S.A.

C. Kennedy, University of Arizona, Tucson, AZ, U.S.A.

H. H. Keyser, University of Hawaii, Paia, HI, U.S.A.

J. Kijne, Leiden University, Leiden, The Netherlands

B. Kirpatrick, University of California, Davis, CA, U.S.A.

D. Klessig, Rutgers The State University of New Jersey, Piscataway, NJ, U.S.A.

J. P. Knox, University of Leeds, Leeds, England, U.S.A.

D. Kobayashi, Rutgers University, New Brunswick, NJ, U.S.A.

K.-H. Kogel, Justus-Liebig-Universitat Gieben, Gieben, Germany

P. E. Kolattukudy, Ohio State University, Columbus, OH, U.S.A.

R. Kolter, Harvard Medical School, Boston, MA, U.S.A.

A. Kondorosi, CNRS, Institut Des Sciences Vegetables, Gif-surYvette Cedex, France
E. Kondorosi, CNRS, Institut Des Sciences Vegetables, Gif-surYvette Cedex, France

H. Kouchi, National Institute of Agrobiological Resources, Tsukuba, Ibaraki, Japan

H. B. Krishnan, University of Missouri-Columbia, Columbia, MO, U.S.A.

J. Kronstad, The University of British Columbia, Vancouver, B.C., Canada

J. Labavitch, University of California, Davis, CA, U.S.A.

G. Lacy, Virginia Polytechnic Institute and State University, Blacksburg, VA, U.S.A.

J. K. Ladha, International Rice Research Institute, Los Banos, Collage, Laguna, Philippines

M. Lagrimini, Ohio State University, Columbus, OH, U.S.A.

Steve Lam, Ciba Geigy Agricultural Biotechnology, Research Triangle Park, NC, U.S.A.

R. Vogeli Lange, Universitat Basel, Basel, Switzerland

M. Law, Ciba Geigy Agricultural Biotechnology, Research Triangle Park, NC, U.S.A.

K. Lawton, Ciba Geigy Agricultural Biotechnology, Research Triangle Park, NC, U.S.A.

D. Lewandowski, University of Florida, Lake Alfred, FL, U.S.A.

C.-H. Liao, Eastern Regional Research Center, Philadelphia, PA, U.S.A.

R. Linderman, USDA, ARS Hortic. Crops. Res. Lab., Corvallis, OR, U.S.A.

S. Lindow, University of California, Berkeley, CA, U.S.A.

G. Lomonossoff, John Innes Centre, Norwich, United Kingdom

S. Long, Stanford University, Stanford, CA, U.S.A.

J. Lorang, Oregon State University, Corvallis, OR, U.S.A.

P. Low, Purdue University, Purdue University, West Lafayette, IN, U.S.A.

R. A. Ludwig, University of California Santa Cruz, Santa Cruz, CA, U.S.A.

B. Lugtenberg, Leiden University, Leiden, The Netherlands

S. Mackenzie, Purdue University, West Lafayette, IN, U.S.A.

R. J. Maier, Johns Hopkins University, Baltimore, MD, U.S.A.

F. Martin, INRA-Nancy, Champenoux, France

G. Martin, Purdue University, West Lafayette, IN, U.S.A.

E. Martinez-Romero, Universidad Nacional Autonoma de Mexico, Cuernavaca, Mor. Mexico

L. Marton, University of South Carolina, Columbia, SC, U.S.A.

G. Marzluf, The Ohio State University, Columbus, OH, U.S.A.

M. A. Mayo, Scottish Crop Research Institute, Invergowie, Dundee, Scotland

M. Mazzola, USDA-ARS, Wenatchee, WA, U.S.A.

S. McCouch, Cornell University, Ithaca, NY, U.S.A.

T. McDermott, Montana State University, Bozeman, MT, U.S.A.

B. McDonald, Texas A\&M University, College Station, TX, U.S.A.

J. L. McEvoy, University of Wisconsin-Madison, Madison, WI, U.S.A.

R. Meeley, TUSC, Pioneer Hi-Bred Intl., Johnston, IA, U.S.A

F. Meins, Jr., Freidrich Meischer Institute, Basel, Switzerland

K. Mendgen, Universitat Konstanz, D-78434 Konstanz, Germany

M. Milgroom, Cornell University, Ithaca, NY, U.S.A.

E. Minami, National Institute of Agrobiological Resources, Kannondai, Tsukuba, Japan

A. Molina, CIBA-Ag. Biotech. Res. Facility, Research Triangle Park, NC, U.S.A.

S. Mongkolsuk, Chulabhorn Research Institute, Lak Si, Bangkok, Thailand 
U. Neuenschwander, Novartis Crop Protection, Inc., Research Triangle Park, NC, U.S.A.

T. Nixon, Pennsylvania State University, University Park, PA, U.S.A.

D. Noel, Marquette University, Milwaukee, WI, U.S.A.

D. Nuss, University of Maryland Biotech Institute, College Park, MD, U.S.A.

M. O'Brien, State University of New York at Buffalo, Buffalo, NY, U.S.A.

R. Oliver, Carlsberg Laboratory, Valby, Denmark

K. Ophel-Keller, SARDI, Hartley Grove Urrbrae, South Africa

L. Owens, USDA, Plant Molecular Lab., Beltsville, MD, U.S.A.

T. Paulitz, Macdonald Campus of McGill University, Anne de Bellevue, Quebec, Canada

N. K. Peters, Norwegian Agricultural University, Norway

D. Phillips, University of California, Davis, CA, U.S.A.

L. S. Pierson III, University of Arizona, Tucson, AZ, U.S.A.

R. K. Poole, The University of Sheffield, Sheffield, United Kingdom

F. Ponz, Centro de Investigaciones y Tecnologia (CIT-INIA), Madrid, Spain

W. A. Powell, SUNY-College of Environmental, Science and Forestry, Syracuse, NY, U.S.A.

U. B. Priefer, RWTH Aachen, Botanische Institut, Aachen, Germany

T. Pryor, CSIRO, Canberra ACT 2601, Australia

N. Quigley, University of Tennessee, Knoxville, TN, U.S.A.

J. Raaijmakers, Washington State University, Pullman, WA, U.S.A.

G. Rao, Pioneer Hi-Bred International, Inc., Johnston, IA, U.S.A.

A. L. N. Rao, University of California, Riverside, CA, U.S.A.

B. Reuhs, University of Georgia, Athens, GA, U.S.A.

K. Richards, Institut de Biologie Moleculaire des Plantes, Strasbourg Cedex, France

G. P. Roberts, University of Wisconsin-Madison, Madison, WI, U.S.A.

B. G. Rolfe, The Australian National University, Canberra, Australia

M. Romantschuk, University of Helsinki, Helsinki, Finland

P. Ronald, University of California, Davis, CA, U.S.A.

M. J. Roossinck, The Samuel Roberts Noble Foundation, Ardmore, OK, U.S.A.

G. Safir, Michigan State University, East Lansing, MI, U.S.A.

J. Salmeron, Novartis Seeds Biotechnology Research Unit, Research Triangle Park, NC, U.S.A.

M. San Francisco, Texas Tech University, Lubbock, TX, U.S.A.

S. Santa Cruz, Scottish Crop Research Institute, Invergowie, Dundee, Scotland

D. Scheel, Institute of Plant Biochemistry, Halle, Germany

J. E. Schoelz, University of Missouri, Columbia, MO, U.S.A.

J. Scott-Craig, Michigan State University, East Lansing, MI, U.S.A.
J. Shaw, Armstrong Laboratory/AOEL, Brooks Air Force Base, TX, U.S.A.

A. Showalter, Ohio State University, Athens, OH, U.S.A.

J. Siedow, Duke University, Raleigh, NC, U.S.A.

A. E. Simon, University of Massachusetts, Amherst, MA, U.S.A.

H. P. Spaink, Leiden University, Leiden, The Netherlands

J. Stanley, John Innes Institute, Norwich, United Kingdom

R. Staples, NYSAES/Cornell University, Geneva, NY, U.S.A.

D. Stenger, Northern Illinois University, Dekalb, IL, U.S.A.

J. Streeter, Ohio State Univ./O.A.R.D.C., Wooster, OH, U.S.A.

J. Sweigard, Stine-Haskell Research Center, Newark, DE, U.S.A.

L. Szabo, University of Minnesota, St. Paul, MN, U.S.A.

K. Szczyglowski, Michigan State University, East Lansing, MI, U.S.A.

J. Takemoto, Utah State University, Logan, UT, U.S.A.

N. J. Talbot, University of Exeter, Exeter, United Kingdom

M. Taliansky, Scottish Crop Research Institute, Invergowie, Dundee, Scotland

D. Tepfer, INRA, F-78026 Versailles Cedex, France

H. Thordal-Christensen, Carnegie Institution of Washington, Stanford, CA, U.S.A.

B. Tyler, University of California, Davis, CA, U.S.A.

S. Ukness, Mol Gen-Ag. Bio.-Ciba Geigy, Research Triangle Park, NC, U.S.A.

B. Valent, El Dupont De Nemours \& Co. Experiment Station, Wilmington, DE, U.S.A.

N. Van Alfen, Texas A\&M University, College Station, TX, U.S.A.

H. van Etten, University of Arizona, Tucson, AZ, U.S.A.

J. A. L. Van Kan, Wageningen Agricultural University, Wageningen, The Netherlands

C. Vance, University of Minnesota, St. Paul, MN, U.S.A.

K. Vandenbosch, Texas A\&M University, College Station, TX, U.S.A.

J. Vanderleyden, Katholieke University Leuven, Heverlee, Belgium

B. Vernooij, Ciba Agricultural Biotechnology, Research Triangle Park, NC, U.S.A.

A. Vivian, University of the West of England, Bristol, United Kingdom

G. Walker, MIT, Cambridge, MA, U.S.A.

S. Wegener, Michigan State University, East Lansing, MI, U.S.A.

M. C. Whalen, San Francisco State University, San Francisco, CA, U.S.A.

M. Willits, Novartis Crop Protection, Research Triangle Park, NC, U.S.A.

J. Xu, Purdue University, West Lafayette, IN, U.S.A.

J. Yoder, University of California, Davis, CA, U.S.A.

S. Young, Dow-Elanco, Indianapolis, IN, U.S.A.

N. Young, University of Minnesota, St. Paul, MN, U.S.A.

M. Zaitlin, Cornell University, Ithaca, NY, U.S.A.

R. Zeyen, University of Minnesota, St. Paul, MN, U.S.A. 